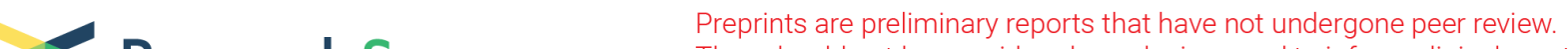 Research Square
or referenced by the media as validated information.
}

\section{Assessment of the Influence of Keratometry on Intraocular Lens Calculation Formulas in Long Axial Length Eyes}

\section{Shengjie Yin}

Joint Shantou International Eye Center of Shantou University and The Chinese University of Hong Kong Chengyao Guo

Joint Shantou International Eye Center of Shantou University and The Chinese University of Hong Kong Kunliang Qiu Joint Shantou International Eye Center of Shantou University and The Chinese University of Hong Kong Tsz Kin Ng Joint Shantou International Eye Center of Shantou University and The Chinese University of Hong Kong Yuancun Li

Joint Shantou International Eye Center of Shantou University and The Chinese University of Hong Kong Yali Du

Joint Shantou International Eye Center of Shantou University and The Chinese University of Hong Kong

\section{Bingyao Chen}

Joint Shantou International Eye Center of Shantou University and The Chinese University of Hong Kong Hongxi Wang

Joint Shantou International Eye Center of Shantou University and The Chinese University of Hong Kong Mingzhi Zhang ( $\nabla$ zmz678096@163.com)

Joint Shantou International Eye Center of Shantou University and the Chinese University of Hong Kong https://orcid.org/0000-0001-9032-7274

\section{Research Article}

Keywords: keratometry, axial length, intraocular lens power calculation, cataract surgery

Posted Date: June 28th, 2021

DOI: https://doi.org/10.21203/rs.3.rs-639671/v1

License: (c) (i) This work is licensed under a Creative Commons Attribution 4.0 International License. Read Full License 


\section{Abstract}

Purpose: Hyperopic surprises tend to occur in axial myopic eyes and other factors including corneal curvature have rarely been analyzed in cataract surgery, especially in eyes with long axial length $(\geq 26.0$ $\mathrm{mm})$. Thus, the purpose of our study was to evaluate the influence of keratometry on four different formulas (SRK/T, Barrett Universal II, Haigis and Olsen) in intraocular lens (IOL) power calculation for long eyes.

Methods: Retrospective case-series. 180 eyes with axial length $(A L) \geq 26.0 \mathrm{~mm}$ were divided into 3 keratometry (K) groups: $K \leq 42.0 \mathrm{D}$ (Flat), $\mathrm{K} \geq 46.0 \mathrm{D}$ (Steep), $42.0<\mathrm{K}<46.0 \mathrm{D}$ (Average). Prediction errors $(\mathrm{PE})$ were compared between different formulas. Multiple regression analysis was performed to investigate factors associated with the PE.

Results: The mean absolute error was higher for all evaluated formulas in Steep group (ranging from 0.66 $\mathrm{D}$ to $1.02 \mathrm{D})$ than the Flat $(0.34 \mathrm{D}$ to $0.67 \mathrm{D})$ and Average groups $(0.40 \mathrm{D}$ to $0.74 \mathrm{D})$. The median absolute errors predicted by Olsen formula were significantly lower than that predicted by Haigis formula ( $0.42 \mathrm{D}$ versus $0.85 \mathrm{D}$ in Steep and 0.29 D versus $0.69 \mathrm{D}$ in Average) in Steep and Average groups $(P=0.012, P<$ 0.001 , respectively). And the Olsen formula demonstrated equal accuracy to the Barrett II formula in Flat and Average groups. The predictability of the SRK/T formula was affected by the AL and K, while the predictability of Olsen and Haigis formulas was affected by the AL only.

Conclusions: Steep cornea has more influence on the accuracy of IOL power calculation than the other corneal shape in long eyes. Overall, both the Olsen and Barrett Universal II formulas are recommended in long eyes with unusual keratometry.

\section{Introduction}

Postoperative refractive accuracy remains to be the major target in cataract surgery. Although new optical biometry and formulas have improved the refractive outcomes of cataract surgery in recent years, intraocular lens $(\mathrm{IOL})$ power calculation in eyes with unusual axial length $(\mathrm{AL})$ and keratometry $(\mathrm{K})$ remains a challenge, and sometimes patients end up with significantly hyperopic or myopic residual ametropia [1, 2]. First, the complicated condition of the fundus, such as posterior staphyloma, in long eyes results in unsatisfactory prediction of the existing IOL calculation formulas [3]. Second, since the effective lens position (ELP) is predicted by the K-value in some conventional IOL formulas, such as the SRK/T, thus the prediction could be significantly affected [4]. Third, as the globe becomes longer the corneal curvature becomes flatter theoretically [5], but the corneal refractive power does not always decrease along with increasing AL in long eyes, which could significantly affect the IOL prediction [6].

A previous study has shown a significant relationship between the corneal asphericity (Q-value) and the error in refraction prediction (a more negative Q-value would lead to a myopic outcome) when using thirdgeneration formulas [7]. However, the variability of the Q-value makes it a poorer predictor of optical quality than the average K-value [8, 9]. Thus most of the IOL power calculation formulas incorporate AL 
and K-value for the ELP prediction, and different formulas could show preferences for eyes with individual ocular features. Adjusting AL or K-value for certain eyes [10,11], optimizing the lens constant in each formula [12], and applying new-generation formulas [13] have been recommended to overcome the challenge under certain situations. Since postoperative hyperopic surprises could always be seen in long eyes, several studies have investigated the relationship between the predictive accuracy of different formulas and the AL, and the SRK/T, Barrett Universal II (Barrett II), Haigis and Olsen formulas have been paid the most attention and been recommended in long eyes [14-16]. However, the influence of keratometry on these formulas in long eyes has seldom been investigated. Therefore, the purpose of the current study was to evaluate the influence of keratometry on four different formulas (SRK/T, Barrett II, Haigis and Olsen) in IOL power calculation for long eyes ( $A L \geq 26.0 \mathrm{~mm}$ ).

\section{Materials And Methods}

This is a retrospective study, and the study protocol was approved by the Ethics Committee for Human Medical Research at the Joint Shantou International Eye Center of Shantou University and the Chinese University of Hong Kong, which was in accordance with the tenets of the Declaration of Helsinki. In total, 15777 electronic medical records of 15777 consecutive patients who underwent phacoemulsification cataract surgery from January 2018 to February 2021 were retrieved. 180 study subjects were finally selected based on the following inclusion criteria: (1) eyes with $A L \geq 26.0 \mathrm{~mm}$; (2) eyes with postoperative corrected distance visual acuity (CDVA) of 6/20 or more within the 1- to 4-month; (3) cataract surgery with "in-the-bag" IOL implantation using 920H IOL model (Rayner Intraocular Lenses Ltd.); and (4) cases of uncomplicated cataract and complete medical records. Patients with traumatic cataract, complicated cataract surgery, pathology affecting the accuracy of biometry calculations (including severe corneal or vitreous opacity, corneal degeneration, retinal detachment and pterygium), previous ocular surgical operation and acquired retinal diseases were not included in this study. Besides, the patients with amblyopia, squint or astigmatism greater than $4.0 \mathrm{D}$ were excluded. If patients underwent bilateral cataract surgery, the eye with better CDVA was selected. If the CDVA of the 2 operated eyes was equal, the first operated eye was selected.

To investigate whether a correlation exists between $\mathrm{K}$-value and postoperative refraction, the studied eyes were divided into three sub-groups based on the K-value (Flat: K $\leq 42.0$ D; Steep: K $\geq 46.0$ D; Average: $42.0<\mathrm{K}<46.0 \mathrm{D}$ ). All patients received phacoemulsification with IOL implantation by experienced surgeons following the standard operation procedure. Biometric parameters including $A L, K$-value, anterior chamber depth (ACD), lens thickness (LT), central corneal thickness (CCT) and white-to-white (WTW), were measured by IOL Master 700 (Carl Zeiss Meditec, Jena, Germany) and OA 2000 (Tomey Corporation, Japan). Prior studies have reported that the IOL Master 700 and OA 2000 showed good agreement in biometric measurements [17, 18].

For each eye, the IOL power was calculated using the Sanders-Retzlaff-Kraff trial (SRK/T), Barrett II, Haigis optimized (Haigis), and Olsen formulas. The lens constants from the user group for laser interference biometry website (ULIB, http://www.augenklinik.uni-wuerzburg.de/ulib) were used for the 
formulas. The prediction error (PE) was calculated as the actual postoperative refraction minus the formula-predicted postoperative refraction. Negative value indicated overcorrection with a tendency of myopic outcomes, whereas positive value indicated undercorrection with a tendency of hyperopic outcomes. The mean absolute error (MAE), median absolute error (MedAE), and the percentages of eyes with refractive errors within $\pm 0.25, \pm 0.50$ and $\pm 1.00 \mathrm{D}$ of the targeted refraction were calculated for each formula.

\section{STATISTICAL ANALYSIS}

All statistical analyses were performed using the commercially available software (IBM SPSS Statistics 21; SPSS Inc., Chicago, IL and R Studio version 1.0.136, R Foundation; Boston, MA). The normality of the data was checked by the Kolmogorov-Smirnov test. Whether the ME for each formula was different from zero was assessed by the one-sample T-test. The differences in the absolute errors among formulas were assessed using the nonparametric Friedman test. In case of significant difference between formulas, post-hoc analysis was conducted using the Wilcoxon signed-rank test with Bonferroni correction. The percentages of eyes within $\pm 0.25, \pm 0.50$ and $\pm 1.00 \mathrm{D}$ of the targeted refraction were compared by the Fisher's exact test. Multiple regression analysis was conducted to evaluate the relationship between the prediction errors and associated factors in all four formulas. Mean (mean \pm standard deviation) and median (median with the inter-quartile range) absolute values were presented. $P<0.05$ was considered to be statistically significant.

\section{Results}

\section{Patients' demographics}

A total of 180 eyes from 180 patients with a mean age of $60.52 \pm 10.28$ were included in the current study. There were 60 males and 120 females, as well as 99 right eyes and 81 left eyes. The mean $\mathrm{K}$ was $44.26 \pm 1.63 \mathrm{D}$, and the mean $\mathrm{AL}$ was $29.35 \pm 2.25 \mathrm{~mm}$. There were 22 eyes in Flat ( $K \leq 42.0 \mathrm{D}), 23$ eyes in Steep $(K \geq 46.0 \mathrm{D})$ and 135 eyes in Average $(42.0<K<46.0 \mathrm{D})$. No significant differences were shown among these three groups considering age, AL, ACD and LT. Table 1 shows the clinical characteristics of the study population.

Accuracy of IOL power calculation formulas in different groups

Statistically significant hyperopic surprises were found with the formulas in Flat and Average groups, except the Olsen formula which showed a slightly myopic surprise in Flat group $(P=0.212)$. In Steep group, only the Haigis formula showed significant hyperopic surprises $(P=0.037)$, and a large standard deviation of the prediction error for all formulas was demonstrated (Table 2 and Fig. 1). In terms of the absolute error, the MAE was higher for all evaluated formulas in Steep group (ranging from $0.66 \mathrm{D}$ to 1.02 D) than the Flat $(0.34 \mathrm{D}$ to $0.67 \mathrm{D})$ and Average groups $(0.40 \mathrm{D}$ to $0.74 \mathrm{D})$. The MedAEs predicted by Olsen formula (0.42 D in Steep and 0.29 D in Average) were significantly lower than that predicted by Haigis formula (0.85 D in Steep and 0.69 D in Average) in Steep and Average groups ( $P=0.012, P<0.001$, 
respectively). And the Olsen formula demonstrated equal accuracy to the Barrett II formula as well as superior to the SRK/T formula in Flat and Average groups.

Figure 2 shows the percentages of eyes within $\pm 0.25 \mathrm{D}, \pm 0.50 \mathrm{D}$ and $\pm 1.00 \mathrm{D}$ of the targeted refraction for four formulas in three groups. The percentages of eyes within $\pm 1.00 \mathrm{D}$ of the targeted refraction were $81.82 \%$ (18/22, SRK/T) to $95.45 \%$ (21/22, Barrett II and Olsen), 56.52\% (13/23, SRK/T) to $78.26 \%(18 / 23$, Barrett II and Olsen), 77.04\% (104/135, Haigis) to $94.07 \%$ (127/135, Barrett II) in Flat, Steep and Average groups, respectively. Of note, as the K-value increased, the predictability for the formulas decreased, especially for the SRK/T and Haigis formulas.

\section{Factors associated with prediction errors of the formulas}

Age, gender and the ocular parameters, such as AL, K, ACD, LT and WTW, were assessed in the multiple regression analysis, which was shown in Table 3. Statistically significant relationships between the PE and the K-value in the SRK/T formula $(\beta=-0.075, P=0.048)$, and the PE and the AL in the Olsen, SRK/T and Haigis formulas were found ( $\beta=0.063 .0 .211$ and 0.091 , respectively). The highest coefficient of determination was demonstrated in the regression equation for the SRK/T formula $\left(\mathrm{PE}_{\mathrm{SRK} / \mathrm{T}}=-4.185+\right.$ $\left.0.211^{\star} A L-0.075 * K, R^{2}=0.344\right)$, followed by the Haigis formula $\left(P E_{\text {Haigis }}=-3.437+0.091 * A L, R^{2}=0.074\right)$. In general, there was stronger relationship between the PE and the ocular parameters, such as AL or Kvalue, for the SRK/T formula. On the other hand, a lower K-value or a longer AL was always associated with a positive PE (hyperopic surprise) and a higher K-value or a shorter AL was always associated with a negative PE (myopic surprise).

\section{Discussion}

Hyperopic surprises tend to occur in axial myopic eyes after cataract surgery, and more attention has been paid to preoperative AL measurement as one of the largest contributors of refractive surprises [19]. Other factors including corneal curvature have rarely been analyzed, especially in this group of patients. Thus, we aimed to evaluate and compare the influence of keratometry on four different formulas in calculating IOL power for highly myopic eyes. Results from the current study indicated that the corneal curvature influenced the refractive outcome of thin-lens IOL calculation formula in long eyes.

Furthermore, the inferior accuracy for all evaluated formulas in steep cornea eyes suggested that steep cornea had more influence on the accuracy of power calculation than the flat cornea and average cornea. Adequate adjustment of formulas according to the K-value for accurate postoperative refraction is required in long eyes with steep cornea.

The postoperative hyperopic surprise in patients with long AL is well known [16, 20]. In agree with previous studies, our regression analysis has shown that this trend can be seen when using the standard formulas, that is to say a longer AL always associated to a positive PE (hyperopic surprise). Interestingly, a significant relationship was found between the K-value and the PE produced by the SRK/T formula in long eyes. It was found that a hyperopic surprise always happened in an oblate cornea and myopic in a 
prolate cornea using the SRK/T, which was consistent with previous studies [7, 21]. A prior study including 18501 single eyes also showed that the reverse tendency was observed in eyes that have flat or steep cornea curvature when using the SRK/T formula as measured by PE [13]. This is not surprising because the paracentral curvature is lower than the central curvature measured by keratometry. However, Zhang et al. [22] observed the PE shifted to hyperopic in prolate corneas and to myopic in oblate corneas in eyes with $\mathrm{AL}>29.0 \mathrm{~mm}$, contrary to our results. Maybe one of the reasons is that unexpected hyperopic outcomes always happen in extremely long eyes using standard formulas, and the other one is that as the globe becomes longer and larger, the axial myopic eyes are demonstrated to have steeper corneas than emmetropic eyes [6], which might lead to the reversed shift to myopic surprises.

In the current study, we found that the Olsen and Barrett II formulas provided superior predictability compared to the SRK/T and Haigis formulas, in terms of the PE, MAE, MedAE and the percentages of eyes within $\pm 0.25 \mathrm{D}, \pm 0.50 \mathrm{D}$ and $\pm 1.00 \mathrm{D}$ of the targeted refraction in all three groups. The Olsen formula using C-constant to predict the ELP precisely is recommended for the eyes with an unusual Kvalue and it has unique advantages compared with SRK/T and Haigis formulas [23]. The SRK/T and Haigis formulas have been proven to perform well in patients with AL of greater than $26.0 \mathrm{~mm}$ [24, 25], however, the two formulas were less accurate in the current study. The inaccurate results of the two formulas might derive from another biometrical factor, the corneal curvature, and this factor may play an important role in the predictive refraction. Olsen et al. [26] reported a significant negative correlation between the prediction error when using the SRK/T formula and the K-values $(r=-0.23, P<0.001)$. Similarly, Reitblat et al. [11] detected that the refractive outcomes of SRK/T formula shifted to myopic $(-0.31 \pm 0.54 \mathrm{D})$ in eyes with a K-value $>46.0 \mathrm{D}$, and to hyperopic $(0.16 \pm 0.31 \mathrm{D})$ in eyes with a K-value < 42.0 D. These results accord closely with ours. lijima et al. [27] compared the predictability using the Barrett II and SRK/T formulas according to the K-values, suggesting that the SRK/T formula was vulnerable to the corneal shape and the Barrett II formula might be more accurate than the SRK/T formula, regardless of whether in eyes with long AL or in eyes with steep or flat cornea, which was consistent with our results. Lately, a study has reported that the Haigis formula presented the most accurate prediction when considering the $\mathrm{AL}$ as well as the corneal power simultaneously, which was contrary to our results showing that the Haigis formula was not as accurate as the other formulas [28]. However, the authors did not compare the new generation formulas such as Olsen or Barrett II in their study, and they excluded extreme eyes with an AL more than $30.0 \mathrm{~mm}$ or a K-reading higher than $49.0 \mathrm{D}$, which may be the reason why they obtained different results from ours. Another study thought the inferior prediction of the Haigis formula in eyes with steep cornea may be due to the inaccuracy of its constants [29]. Thus adjustment of these constants may improve the predictability of the Haigis formula.

In the present study, we found that the commonly used formula SRK/T was less accurate than the Olsen or Barrett II formula, especially in eyes with steep cornea. Sheard et al. [30] proposed to use the T2 formula as a substitution for the SRK/T formula. Moreover, Reitblat et al. [11] suggested to develop equations for optimizing the K-values based on the linear regression analysis when using the SRK/T formula. In addition, Eom et al. [31] employed different lens constants (higher for flat corneas and lower 
for steep corneas) to improve the predictive refraction with the SRK/T formula. Yet, these modified methods are derived from the eyes with normal AL. Whether these methods could perform well in eyes with long AL should be further investigated.

There are several limitations in this study. First, the relatively small sample size in the Flat and Steep subgroups might be insufficient to perform a meaningful subgroup analysis for comparing formula accuracy. A study of a larger sample size is needed to validate the accuracy of prediction among these formulas. Second, the ME was not equal to zero by changing the lens constant personality for each formula [32]. However, a previous study has reported that optimized lens constant showed no significant refractive advantages over the ULIB constant in the eyes with long $A L$, and suggested that it is not necessary to optimize lens constants for long eyes alone [33]. Third, different surgeons might result in bias from their differences in operation. However, the refractive outcomes in cataract surgery in a single center would less likely be affected by the variations among surgeons [34]. Accordingly, we assume that the impact of surgeons on the outcomes could be clinically negligible in the current case series.

In summary, keratometry influences the accuracy of IOL power calculation, and steep cornea has more influence than the other corneal shape in long eyes. Overall, both the Olsen and Barrett Universal II formulas are suitable in long eyes with unusual keratometry. The commonly used SRK/T formula, which tends to myopic in steep cornea and to hyperopic in flat cornea eyes, is not recommended and should be adjusted in this situation.

\section{Declarations}

\section{Funding:}

This work was supported by the Grant for Key Disciplinary Project of Clinical Medicine under the Guangdong High-level University Development Program (No. 58, Shanfu Section [2020]), China.

Conflicts of interest: The authors have no relevant financial or non-financial interest to disclose.

\section{Authors' contributions:}

(I) Conceptualization and design: Shengjie Yin, Kunliang Qiu; (II) Methodology: Shengjie Yin, Kunliang Qiu, Chengyao Guo; (III) Formal analysis and investigation: Chengyao Guo, Yuancun Li; (IV) Writingoriginal draft preparation: Chengyao Guo; (V) Writing-review and editing: Chengyao Guo, Kunliang Qiu, Tsz Kin Ng; (VI) Resources: Shengjie Yin, Yali Du, Bingyao Chen, Hongxi Wang; (VII) Supervision: Mingzhi Zhang; (VIII) Funding acquisition: Shengjie Yin.

\section{Ethics approval}

The study was conducted in accordance with the Declaration of Helsinki (as revised in 2013). The study was approved by the Ethics Committee for Human Medical Research at the Joint Shantou International 
Eye Center of Shantou University and the Chinese University of Hong Kong (No. 58, Shanfu Section [2020]).

\section{Consent to participate:}

Individual consent for this retrospective analysis was waived.

\section{Consent for publication:}

Not available.

\section{Acknowledgment}

The authors would like to express their deepest gratitude to all the participants in this study. This work was supported by the Grant for Key Disciplinary Project of Clinical Medicine under the Guangdong Highlevel University Development Program (No. 58, Shanfu Section [2020]), China.

\section{References}

[1] Doshi D, Limdi P, Parekh N, et al (2017) A Comparative Study to Assess the Predictability of Different IOL Power Calculation Formulas in Eyes of Short and Long Axial Length. J Clin Diagn Res. 11(1): C1-C4. doi: $10.7860 / J C D R / 2017 / 22095.9136$

[2] Lundstrom M, Barry P, Henry Y, et al (2012) Evidence-based guidelines for cataract surgery: guidelines based on data in the European Registry of Quality Outcomes for Cataract and Refractive Surgery database. J Cataract Refract Surg. 38(6): 1086-1093. doi: 10.1016/j.jcrs.2012.03.006

[3] McBrien N A, Gentle A (2003) Role of the sclera in the development and pathological complications of myopia. PROG RETIN EYE RES. 22(3): 307-338. doi: 10.1016/s1350-9462(02)00063-0

[4] Wang L, Shirayama M, Ma X J, et al (2011) Optimizing intraocular lens power calculations in eyes with axial lengths above $25.0 \mathrm{~mm}$. J Cataract Refract Surg. 37(11): 2018-2027. doi:

10.1016/j.jcrs.2011.05.042

[5] Hoffer K J, Savini G (2017) Effect of Gender and Race on Ocular Biometry. Int Ophthalmol Clin. 57(3): 137-142. doi: 10.1097/II0.0000000000000180

[6] Carney L G, Mainstone J C, Henderson B A (1997) Corneal topography and myopia. A cross-sectional study. Invest Ophthalmol Vis Sci. 38(2): 311-320. doi

[7] Savini G, Hoffer K J, Barboni P (2015) Influence of corneal asphericity on the refractive outcome of intraocular lens implantation in cataract surgery. J Cataract Refract Surg. 41(4): 785-789. doi:

10.1016/j.jcrs.2014.07.035 
[8] Arba M S (2015) Influence of corneal asphericity on refractive outcomes after cataract surgery. J Cataract Refract Surg. 41(8): 1797-1798. doi: 10.1016/j.jcrs.2015.05.021

[9] Hoffer K J, Aramberri J, Haigis W, et al (2015) Protocols for studies of intraocular lens formula accuracy. AM J OPHTHALMOL. 160(3): 403-405. doi: 10.1016/j.ajo.2015.05.029

[10] Zhang J, Tan X, Wang W, et al (2020) Effect of Axial Length Adjustment Methods on Intraocular Lens Power Calculation in Highly Myopic Eyes. AM J OPHTHALMOL. 214: 110-118. doi:

10.1016/j.ajo.2020.02.023

[11] Reitblat O, Levy A, Kleinmann G, et al (2017) Intraocular lens power calculation for eyes with high and low average keratometry readings: Comparison between various formulas. J Cataract Refract Surg. 43(9): 1149-1156. doi: 10.1016/j.jcrs.2017.06.036

[12] Hoffer K J, Savini G (2020) Update on Intraocular Lens Power Calculation Study Protocols: The Better Way to Design and Report Clinical Trials. OPHTHALMOLOGY. doi: 10.1016/j.ophtha.2020.07.005

[13] Melles R B, Holladay J T, Chang W J (2018) Accuracy of Intraocular Lens Calculation Formulas. OPHTHALMOLOGY. 125(2): 169-178. doi: 10.1016/j.ophtha.2017.08.027

[14] Cheng H, Wang L, Kane J X, et al(2020)Accuracy of Artificial Intelligence Formulas and Axial Length Adjustments for Highly Myopic Eyes. AM J OPHTHALMOL. 223: 100-107. doi:

10.1016/j.ajo.2020.09.019

[15] Rong X, He W, Zhu Q, et al (2019) Intraocular lens power calculation in eyes with extreme myopia: Comparison of Barrett Universal II, Haigis, and Olsen formulas. J Cataract Refract Surg. 45(6): 732-737. doi: $10.1016 /$ j.jcrs.2018.12.025

[16] Bang S, Edell E, Yu Q, et al (2011) Accuracy of Intraocular Lens Calculations Using the IOLMaster in Eyes with Long Axial Length and a Comparison of Various Formulas. OPHTHALMOLOGY. 118(3): 503506. doi: 10.1016/j.ophtha.2010.07.008

[17] Liao X, Peng Y, Liu B, et al (2020) Agreement of ocular biometric measurements in young healthy eyes between IOLMaster 700 and OA-2000. Sci Rep. 10(1): 3134. doi: 10.1038/s41598-020-59919-y

[18] Hua Y, Qiu W, Xiao Q, et al (2018) Precision (repeatability and reproducibility) of ocular parameters obtained by the Tomey OA-2000 biometer compared to the IOLMaster in healthy eyes. PLOS ONE. 13(2): e193023. doi: 10.1371/journal.pone. 0193023

[19] Kane J X, Melles R B (2020) Intraocular lens formula comparison in axial hyperopia with a highpower intraocular lens of 30 or more diopter. J Cataract Refract Surg. doi:

$10.1097 /$ j.jcrs. 0000000000000235 
[20] Wan K H, Lam T C H, Yu M C V, et al (2019) Accuracy and Precision of Intraocular Lens Calculations Using the New Hill-RBF Version 2.0 in Eyes With High Axial Myopia. AM J OPHTHALMOL. 205: 66-73. doi: 10.1016/j.ajo.2019.04.019

[21] Minami K, Kataoka Y, Matsunaga J, et al (2012) Ray-tracing intraocular lens power calculation using anterior segment optical coherence tomography measurements. J Cataract Refract Surg. 38(10): 17581763. doi: $10.1016 / j . j c r s .2012 .05 .035$

[22] Zhang Z, Miao Y, Fang X, et al (2018) Accuracy of the Haigis and SRK/T Formulas in Eyes Longer than $29.0 \mathrm{~mm}$ and the Influence of Central Corneal Keratometry Reading. CURR EYE RES. 43(11): 13161321. doi: $10.1080 / 02713683.2018 .1488265$

[23] Oh J H, Kim S H, Chuck R S, et al (2014) Evaluation of the Pentacam ray tracing method for the measurement of central corneal power after myopic photorefractive keratectomy. CORNEA. 33(3): 261265. doi: $10.1097 /$ ICO.0000000000000034

[24] Abulafia A, Barrett G D, Rotenberg M, et al (2015) Intraocular lens power calculation for eyes with an axial length greater than $26.0 \mathrm{~mm}$ : comparison of formulas and methods. J Cataract Refract Surg. 41(3): 548-556. doi: 10.1016/j.jcrs.2014.06.033

[25] Voytsekhivskyy O V (2018) Development and Clinical Accuracy of a New Intraocular Lens Power Formula (VRF) Compared to Other Formulas. AM J OPHTHALMOL. 185: 56-67. doi: 10.1016/j.ajo.2017.10.020

[26] Olsen T, Hoffmann P (2014) C constant: new concept for ray tracing-assisted intraocular lens power calculation. J Cataract Refract Surg. 40(5): 764-773. doi: 10.1016/j.jcrs.2013.10.037

[27] lijima K, Kamiya K, lida Y, et al (2020) Comparison of Predictability Using Barrett Universal II and SRK/T Formulas according to Keratometry. J OPHTHALMOL. 2020: 7625725. doi:

$10.1155 / 2020 / 7625725$

[28] Roggla V, Langenbucher A, Leydolt C, et al (2020) Accuracy of common IOL power formulas in 611 eyes based on axial length and corneal power ranges. $\mathrm{Br} \mathrm{J}$ Ophthalmol. doi: 10.1136/bjophthalmol-2020315882

[29] Faramarzi A, Aghajani A, Ghiasian L (2017) Accuracy of Various Intraocular Lens Power Calculation Formulas in Steep Corneas. J Ophthalmic Vis Res. 12(4): 385-389. doi: 10.4103/jovr.jovr_20_17

[30] Sheard R M, Smith G T, Cooke D L (2010) Improving the prediction accuracy of the SRK/T formula: the T2 formula. J Cataract Refract Surg. 36(11): 1829-1834. doi: 10.1016/j.jcrs.2010.05.031

[31] Eom Y, Kang S Y, Song J S, et al (2013) Use of corneal power-specific constants to improve the accuracy of the SRK/T formula. OPHTHALMOLOGY. 120(3): 477-481. doi:

10.1016/j.ophtha.2012.09.008

Page $10 / 14$ 
[32] Abulafia A, Barrett G D, Koch D D, et al (2016) Protocols for Studies of Intraocular Lens Formula Accuracy. AM J OPHTHALMOL. 164: 149-150. doi: 10.1016/j.ajo.2016.01.010

[33] Zhang J Q, Zou X Y, Zheng D Y, et al (2019) Effect of lens constants optimization on the accuracy of intraocular lens power calculation formulas for highly myopic eyes. Int J Ophthalmol. 12(6): 943-948. doi: $10.18240 /$ ijo.2019.06.10

[34] Aristodemou P, Knox C N, Sparrow J M, et al (2011) Intraocular lens formula constant optimization and partial coherence interferometry biometry: Refractive outcomes in 8108 eyes after cataract surgery. $J$ Cataract Refract Surg. 37(1): 50-62. doi: 10.1016/j.jcrs.2010.07.037

\section{Tables}

Table 1

Characteristics of Eyes in the Study.

\begin{tabular}{|c|c|c|c|}
\hline Parameter & $\begin{array}{l}\text { Flat } \\
(K \leq 42.0 D, n=22)\end{array}$ & $\begin{array}{l}\text { Steep } \\
(K \geq 46.0 D, n=23)\end{array}$ & $\begin{array}{l}\text { Average } \\
(42.0 \mathrm{D}<\mathrm{K}<46.0 \mathrm{D}, \mathrm{n}=135)\end{array}$ \\
\hline Age & $64.77 \pm 10.83$ & $60.74 \pm 11.21$ & $59.79 \pm 9.94$ \\
\hline $\begin{array}{l}\text { Gender, n (\%) } \\
\text { Male } \\
\text { Female }\end{array}$ & $\begin{array}{l}12(54.55 \%) \\
10(45.45 \%)\end{array}$ & $\begin{array}{l}2(8.70 \%) \\
21(91.30 \%)\end{array}$ & $\begin{array}{l}46(34.07 \%) \\
89(65.93 \%)\end{array}$ \\
\hline $\begin{array}{l}\text { Eye, n (\%) } \\
\text { Right } \\
\text { Left }\end{array}$ & $\begin{array}{l}12(54.55 \%) \\
10(45.45 \%)\end{array}$ & $\begin{array}{l}14(60.87 \%) \\
9(39.13 \%)\end{array}$ & $\begin{array}{l}72(53.33 \%) \\
63(46.67 \%)\end{array}$ \\
\hline $\begin{array}{l}\text { Average K (D) } \\
\text { K1 (D) } \\
\text { K2 (D) }\end{array}$ & $\begin{array}{l}41.49 \pm 0.47 \\
40.93 \pm 0.61 \\
42.07 \pm 0.71\end{array}$ & $\begin{array}{l}46.92 \pm 0.72 \\
46.15 \pm 0.68 \\
47.69 \pm 0.92\end{array}$ & $\begin{array}{l}44.25 \pm 0.97 \\
43.69 \pm 1.05 \\
44.81 \pm 1.00\end{array}$ \\
\hline $\mathrm{AL}(\mathrm{mm})$ & $28.68 \pm 2.58$ & $28.78 \pm 2.04$ & $29.56 \pm 2.21$ \\
\hline ACD (mm) & $3.38 \pm 0.31$ & $3.42 \pm 0.41$ & $3.51 \pm 0.44$ \\
\hline LT (mm) & $4.57 \pm 0.46$ & $4.31 \pm 0.43$ & $4.44 \pm 0.43$ \\
\hline WTW (mm) & $12.04 \pm 0.39$ & $11.31 \pm 0.28$ & $11.62 \pm 0.41$ \\
\hline
\end{tabular}


Table 2

Refractive prediction error, mean absolute error and median absolute error produced by each formula.

\begin{tabular}{|c|c|c|c|c|}
\hline \multirow{6}{*}{$\begin{array}{l}\text { Flat } \\
(\mathrm{K} \leq 42.0 \mathrm{D})\end{array}$} & Formula & $\mathrm{ME} \pm \mathrm{SD}(\mathrm{D})$ & $\mathrm{MAE} \pm \mathrm{SD}(\mathrm{D})$ & $\operatorname{MedAE}(\mathrm{IQR})(\mathrm{D})$ \\
\hline & Olsen & $-0.12 \pm 0.44$ & $0.34 \pm 0.29$ & $0.22(0.36)$ \\
\hline & Barrett II & $0.24 \pm 0.43$ & $0.41 \pm 0.27$ & $0.33(0.39)$ \\
\hline & $\mathrm{SRK} / \mathrm{T}$ & $0.65 \pm 0.45$ & $0.67 \pm 0.41$ & $0.71(0.51)$ \\
\hline & Haigis & $0.57 \pm 0.45$ & $0.63 \pm 0.37$ & $0.68(0.52)$ \\
\hline & $P$ value & - & $<0.001$ & $<0.001$ \\
\hline \multirow{6}{*}{$\begin{array}{l}\text { Steep } \\
(K \geq 46.0 D)\end{array}$} & Formula & $\mathrm{ME} \pm \mathrm{SD}(\mathrm{D})$ & $M A E \pm S D(D)$ & MedAE (IQR)(D) \\
\hline & Olsen & $-0.10 \pm 1.07$ & $0.66 \pm 0.83$ & $0.42(0.61)$ \\
\hline & Barrett II & $-0.06 \pm 1.14$ & $0.73 \pm 0.86$ & $0.31(0.72)$ \\
\hline & $\mathrm{SRK} / \mathrm{T}$ & $0.09 \pm 1.20$ & $0.94 \pm 0.72$ & $0.74(1.16)$ \\
\hline & Haigis & $0.50 \pm 1.09$ & $1.02 \pm 0.60$ & $0.85(0.84)$ \\
\hline & $P$ value & - & 0.015 & 0.015 \\
\hline \multirow{6}{*}{$\begin{array}{l}\text { Average } \\
(42.0 \mathrm{D}<\mathrm{K}<46.0 \mathrm{D})\end{array}$} & Formula & $\mathrm{ME} \pm \mathrm{SD}(\mathrm{D})$ & $M A E \pm S D(D)$ & MedAE (IQR)(D) \\
\hline & Olsen & $0.08 \pm 0.57$ & $0.41 \pm 0.40$ & $0.29(0.39)$ \\
\hline & Barrett II & $0.21 \pm 0.52$ & $0.40 \pm 0.39$ & $0.30(0.41)$ \\
\hline & $\mathrm{SRK} / \mathrm{T}$ & $0.57 \pm 0.76$ & $0.70 \pm 0.63$ & $0.60(0.68)$ \\
\hline & Haigis & $0.70 \pm 0.57$ & $0.74 \pm 0.51$ & $0.69(0.59)$ \\
\hline & $P$ value & - & $<0.001$ & $<0.001$ \\
\hline
\end{tabular}


Table 3

Associated factors for intraocular lens power prediction among formulas.

\begin{tabular}{|lllll|}
\hline & Olsen & Barrett Universal II & SRK/T & Haigis \\
\hline & $\beta$ & $\beta$ & $\beta$ & $\beta$ \\
\hline Age & 0.006 & 0.005 & 0.006 & 0.006 \\
\hline Gender-male & -0.096 & -0.084 & -0.074 & -0.085 \\
\hline Axial length (mm) & $0.063^{\star \star}$ & 0.028 & $0.211^{\star \star \star}$ & $0.091^{\star \star \star}$ \\
\hline Keratometry (D) & 0.021 & -0.032 & $-0.075^{\star}$ & 0.011 \\
\hline Anterior chamber depth $(\mathrm{mm})$ & -0.021 & 0.145 & 0.187 & 0.072 \\
\hline Lens thickness (mm) & -0.074 & 0.065 & 0.032 & 0.076 \\
\hline White to white (mm) & 0.053 & 0.000 & 0.061 & 0.004 \\
\hline Adjusted R-squared & 0.013 & -0.011 & 0.344 & 0.074 \\
\hline P-value & 0.231 & 0.661 & 0.000 & 0.005 \\
\hline
\end{tabular}

\section{Figures}

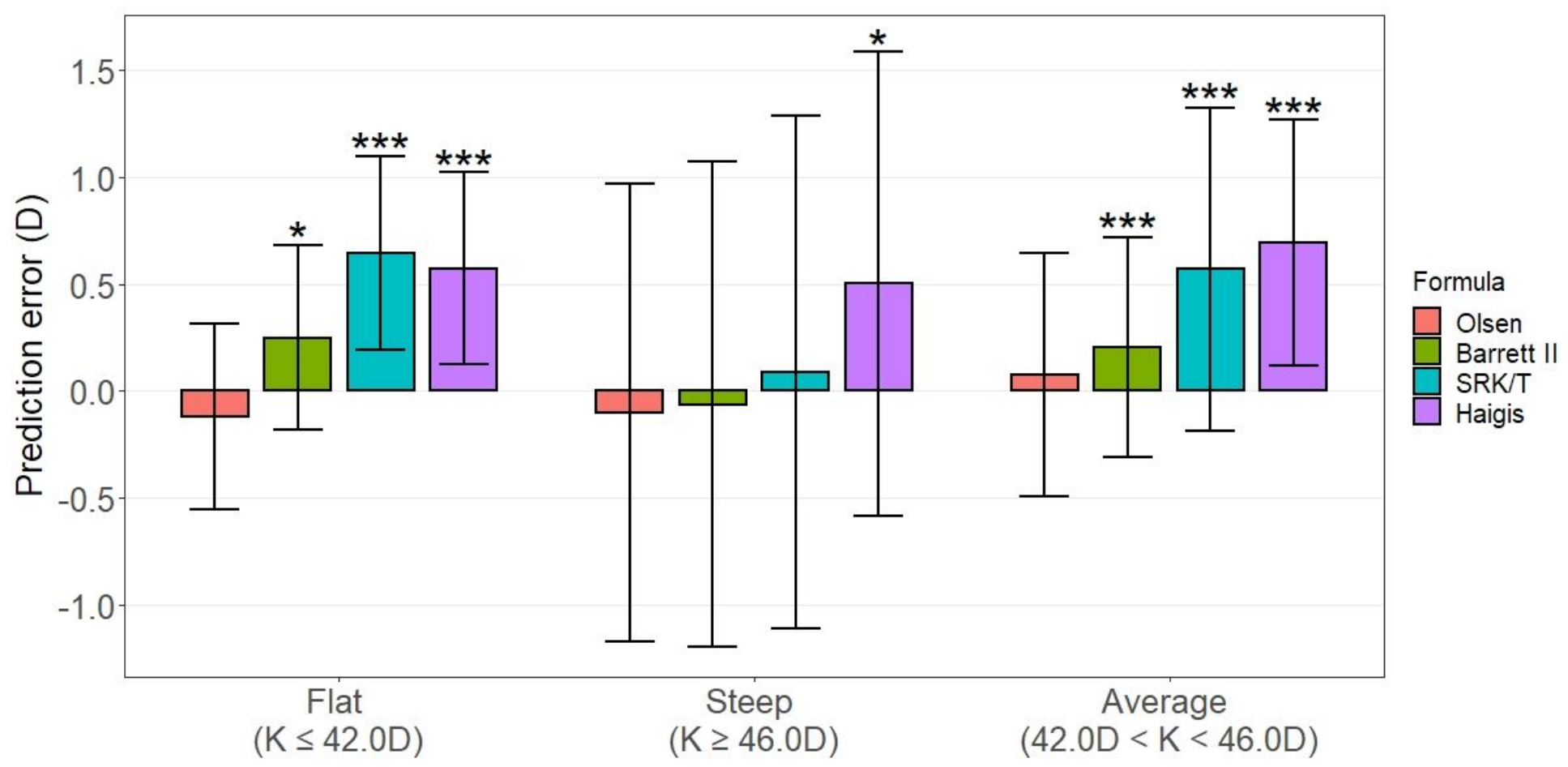

Figure 1 
The distribution of prediction errors for four formulas in each group The tops of the bars represent the mean prediction errors, and the whiskers represent the standard deviations. $P$ values were calculated using the one-sample T-test, values $<0.05$ were considered statistically significant. $*$ : $P<0.05$. $* *$ : $P<$ 0.01 . ${ }^{\star *}: \mathrm{P}<0.001$. Note that hyperopic prediction errors are indicated by positive values. (Barrett II = Barrett Universal II)

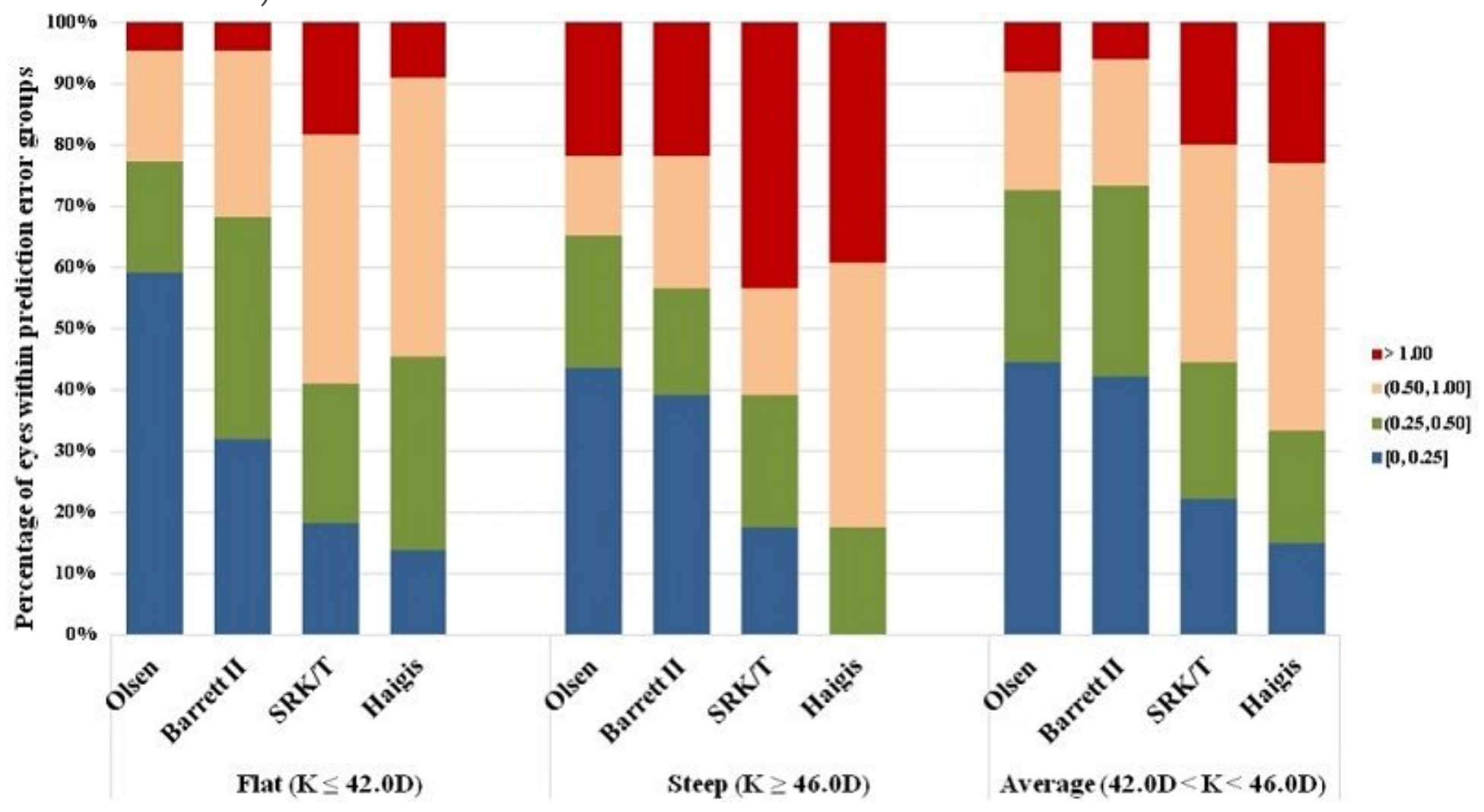

\section{Figure 2}

Percentage of eyes within $\pm 0.25 \mathrm{D}, \pm 0.50 \mathrm{D}$ and $\pm 1.00 \mathrm{D}$ of the target refraction for each formula in each group (Barrett II= Barrett Universal II) 